\title{
Recherche précompétitive, une mise en synergie des ressources publiques et privées : pour quels objectifs, comment et dans quel cadre juridique?
}

\author{
Gilles Vassal ${ }^{1}$, Carole Neves ${ }^{2}$, Olivier Arnaud ${ }^{3}$, Yannick Pletan ${ }^{4}$ et les participants de la Table Ronde $N^{\circ} 1$ \\ de Giens XXVI \\ 1 Direction de la Recherche Clinique, Institut Gustave Roussy, Villejuif, France \\ 2 Sanofi-Aventis, Partenariats France R\&D, Paris, France \\ 3 Inserm Transfert, Paris, France \\ 4 Roche, Neuilly sur Seine, France
}

Texte reçu le 31 mai 2011 ; accepté le 27 juin 2011

\begin{abstract}
Mots clés :
médicaments ;

interdisciplinarité ;

partage ;

propriété intellectuelle

Résumé - L'objectif de la Table Ronde était d'aborder la Recherche Pré-compétitive pour la première fois lors des Rencontres de Giens. Les participants venaient de l'industrie du médicament, des biotech, de l'industrie du dispositif, de l'académie, des services et sociétés de valorisation, des ministères, du monde de la recherche clinique et fondamentale. Ils ont fait le constat des changements profonds dans la connaissance des maladies et dans le développement des médicaments. À l'aide d'exemples dans le monde de la Santé et en dehors, ils ont identifié les principes, les enjeux, les facteurs de succès et de risque de la recherche précompétitive. Les particularités de la gestion de la propriété intellectuelle ont été plus particulièrement étudiées. La Table Ronde a conclu sur les principes de la recherche précompétitive en Santé, une révolution culturelle nécessaire. C'est développer une recherche en partenariat fondée sur des principes nouveaux : i) comprendre et partager; ii) intégrer la complexité du vivant ; iii) agir dans l'interdisciplinarité.
\end{abstract}

\section{Le contexte}

Force est de constater que le modèle classique de développement des médicaments doit être repensé en raison de sa productivité trop faible et des taux d'attrition trop élevés. Dans une analyse récente de l'innovation pharmaceutique au cours des 60 dernières années, Munos indique que plus de 1200 nouveaux médicaments ont été approuvés par la Food and drug administration (FDA) depuis 1950. ${ }^{[1]}$ Cependant, l'industrie pharmaceutique ne génère pas plus de nouveaux médicaments aujourd'hui qu'il y a 60 ans, alors que la connaissance des maladies (et de leur complexité) a profondément changé, ouvrant autant d'opportunités pour des thérapies innovantes. En 2009, l'Agence européenne n'a délivré une autorisation de mise sur le marché que pour 29 nouvelles substances actives. ${ }^{[2]}$ Certains domaines comme la maladie d'Alzheimer et les désordres neuropsychiatriques constituent un challenge

\footnotetext{
* Pour la liste des participants, voir en fin d'article
}

thérapeutique majeur alors qu'il n'y a pas actuellement de médicaments efficaces. L'oncologie fait face à une diversification majeure des molécules thérapeutiques et de leurs cibles (avec plus de 800 anticancéreux en développement) et à la nécessité de développer les biomarqueurs indispensables pour prescrire ces médicaments aux patients qui vont réellement en bénéficier.

Les modèles sont en effet insuffisamment prédictifs de la toxicité ou de l'efficacité. Des médicaments échouent en phase III. Le torcetrapib est un inhibiteur d'une protéine de transfert des esters de cholestérol agissant comme un modulateur dans le traitement des dyslipidémies. Son développement a été arrêté en décembre 2006 lorsque l'étude de phase III ILLUMINATE a montré un excès d'événements cardiovasculaires et de décès inexpliqués. ${ }^{[3]}$ Cette étude avait inclus plus de 15000 patients. Le programme de développement représentait plus de $800 \mathrm{M} \$$ d'investissement. ${ }^{[4]}$ D'autres produits échouent pour manque d'efficacité en phase II ou III. C'est le cas de plusieurs anticorps monoclonaux dirigés 
contre le récepteur à l'insuline (IGF1R) dans différentes pathologies malignes. Les résultats négatifs des études ne sont pas suffisamment exploités et peuvent rester des données mortes alors qu'elles pourraient être riches d'enseignement, lorsqu'elles sont réunies, pour comprendre les raisons des échecs.

Or les investissements en recherche et développement (R\&D) sont importants dans le domaine de la Santé, de 11 à $16 \%$ en moyenne alors qu'ils ne représentent que $5 \%$ au maximum et souvent beaucoup moins dans les autres secteurs industriels. Plusieurs experts de l'industrie pharmaceutique s'accordent à penser que la productivité du modèle de R\&D actuel montre ces limites dans le contexte des contraintes budgétaires qui pèsent sur les systèmes de santé, des pertes de revenus liées à l'expiration des brevets, des exigences réglementaires renforcées, en particulier en termes de balance bénéfice-risque et de vigilance, et de la segmentation du marché qu'imposera la médecine personnalisée. ${ }^{[5-7]}$ Le nombre toujours limité de médicaments mis sur le marché par an (25 seulement en 2010 selon la FDA) est un enjeu pour la société (de nombreuses maladies restent sans solutions thérapeutiques) et un risque pour les industries de la Santé (limitation du retour possible sur investissement). Seulement une molécule sur trois atteignant l'autorisation de mise sur le marché (AMM) retourne son investissement de R\&D. ${ }^{[8]}$

Le secteur du développement des médicaments par les industries pharmaceutiques se complexifie et s'enrichit du développement des biomarqueurs et de la nécessaire collaboration avec les industries du diagnostic. Mais l'accès aux ressources biologiques est difficile et l'accès aux patients devient de plus en plus délicat lorsque les études s'adressent à des sous-populations de malades.

Dans le cas des dispositifs médicaux, les technologies sont difficiles à protéger. C'est l'avance technologique qui fait la valeur pour les entreprises qui les développent. Il s'agit là donc moins d'envisager une recherche pré-compétitive avec des concurrents que de trouver des partenaires industriels aux compétences complémentaires avec qui développer des partenariats.

Le contexte de la recherche biomédicale et scientifique dans le milieu académique (établissements publics de science et technologie, universités, établissements hospitaliers) impose aussi de faire évoluer non seulement les partenariats avec les industriels mais aussi les collaborations entre partenaires publics. Comprendre les maladies, leurs mécanismes moléculaires et faciliter l'accès des patients aux innovations thérapeutiques nécessite de plus en plus l'accès à une masse critique d'information, imposant le partage et la mutualisation des données (médicales, biologiques...), l'accès à des plateformes technologiques et des ressources biologiques partagées et une augmentation du nombre de patients qui se voient proposer de participer à une recherche. Ainsi, le Plan Cancer 2009-2013 fixe un objectif de $10 \%$ des patients traités pour un cancer qui participent à la recherche clinique.
La recherche clinique constitue aussi une des mesures phares du Plan Alzheimer 2008-2012, soutenue par le Programme hospitalier de recherche clinique.

\section{L'enjeu}

Le séquençage du génome humain et le développement des techniques de biologie à haut débit (génomique, protéomique, séquençage) transforment la connaissance des maladies et du rôle du patrimoine génétique, créant autant de ressources innovantes pour de nouvelles cibles thérapeutiques, de nouveaux médicaments, de nouveaux tests diagnostiques.

Il est nécessaire d'intégrer la complexité du vivant pour développer la médecine personnalisée : the right drug(s), for the right patient at the right moment.

- C'est un enjeu pour les patients souffrant de maladies graves mettant en jeu leur pronostic vital ou entrainant une dégradation majeure de leurs conditions de vie.

- C'est un enjeu pour les industriels soucieux d'optimiser le développement des médicaments, par des processus plus rapides et moins onéreux pour des produits plus efficaces et mieux tolérés.

- C'est un enjeu pour les pouvoirs publics et les institutionnels pour améliorer la prise en charge, en particulier des affections graves, réserver les médicaments onéreux aux patients susceptibles d'en bénéficier et mieux contrôler les dépenses de santé.

- C'est un enjeu pour les équipes académiques de recherche pour prendre en compte l'optique «applicative» de leur recherche via des partenariats, tout en conservant leur indépendance et la composante cognitive de leur recherche.

Il s'agit de changer d'échelle dans l'utilisation des données intégrées et de développer un nouveau paradigme pour l'innovation, le développement, l'enregistrement et la prescription des médicaments.

C'est in fine avoir des algorithmes décisionnels utilisant les informations biologiques et génétiques pour prescrire au mieux le meilleur traitement possible.

\section{Pourquoi la recherche précompétitive?}

- Parce que nul ne peut résoudre seul les problèmes actuels et futurs du développement des médicaments.

- Parce qu'il faut partager, mutualiser :

- le savoir, le savoir faire, les ressources, les outils, les modèles ;

- les risques et les coûts. 
- Parce qu'il faut une approche interdisciplinaire, impliquant et synergisant de nouveaux métiers, de nouvelles compétences (modélisation, intégration mathématique, sciences humaines et sociales pour une approche holistique de l'homme).

L'ouverture du champ du développement des médicaments à de nouvelles compétences apparait comme essentielle et incontournable pour prendre en compte la complexité du vivant et des maladies.

\section{Des exemples à succès de recherche précompétitive}

Les industriels Hors Santé ont déjà démontré dans plusieurs domaines le succès que peut apporter une recherche précompétitive. C'est le cas du consortium de recherche précompétitive en traitement et transformation des substances minérales (COREM) créé en septembre 1999, ${ }^{[9]}$ de l'industrie des semi-conducteurs (Sematech) dans les années $1990^{[10]}$ ou du partenariat entre $T I$ Food and Nutrition, un institut néerlandais, avec cinq géants de l'industrie de l'alimentation (Nestlé, Kellogg, Danone, Christian Hansen et Fromageries Bel) pour conduire une recherche fondamentale pré-compétitive sur la santé intestinale et les effets des probiotiques. ${ }^{[11]}$

Dans l'industrie de la Santé, les initiatives sont plus récentes.

En 2007, la Commission Européenne a lancé, en association avec l'European federation of pharmaceutical industries and associations (EFPIA) l'initiative technologique conjointe Innovative medicine initiative (IMI) pour soutenir le développement de nouvelles connaissances, de nouveaux outils et de nouvelles méthodes afin de découvrir, développer et distribuer plus rapidement des médicaments plus appropriés et plus sûrs. La priorité des projets IMI est le développement d'outils précompétitifs d'aide à la découverte de médicaments dans le cadre d'un consortium européen entre académiques et entreprises de tailles diverses. La protection de la propriété intellectuelle est essentielle sur toute la durée de ces projets (cinq ans). Trois appels à projets, dans tous les domaines du médicament, ont déjà eu lieu et le quatrième est en cours d'élaboration.

Un des premiers projets IMI financés par cette initiative est le projet PharmaCog que Olivier Blin coordonne au sein de l'Université de la Méditerranée avec le Dr. Elaine Irving de GlaxoSmithKline, partenaire EFPIA. ${ }^{[12]}$ PharmaCog a été évalué comme le meilleur projet soumis sur une vingtaine de projets concurrents sur les maladies neurodégénératives en 2009. Il a officiellement débuté le $1^{\text {er }}$ Janvier 2010 pour une durée de cinq ans. PharmaCog est un exemple de collaboration publique/privée réussie qui s'inscrit parfaitement dans la lignée du plan national Alzheimer. Avec un cofinancement de $9 \mathrm{M} €$ de l'IMI et de
$13 \mathrm{M} €$ des grands groupes de l'industrie pharmaceutique, PharmaCog a été un des projets les plus ambitieux jamais soumis à financement et dédié à lutter contre la Maladie d'Alzheimer au niveau Européen. De plus, c'est également le premier projet regroupant, autour de 30 participants venant de 9 pays Européens, les principaux acteurs du secteur de la recherche pharmaceutique : 12 partenaires académiques, 12 grands groupes pharmaceutiques, 5 petites et moyennes entreprises (PME) et 1 association de patients, Alzheimer Europe, auxquels s'ajoute l'Agence européenne du médicament (EMA) en tant que partenaire associé.

Le concept de PharmaCog repose sur une analyse des échecs antérieurs. L'approche innovante de PharmaCog consiste à améliorer la prédictivité des molécules en développement pour la maladie d'Alzheimer en :

- ciblant précisément le potentiel d'une molécule et en diminuant leur temps de développement;

- améliorant l'efficience des études cliniques et en diminuant leurs durées et leurs coûts ;

- accélérant à long terme la mise sur le marché de nouveaux médicaments prometteurs.

Le troisième appel à projets IMI a élargi les thèmes à ceux de l'hépatotoxicité, l'immunogénicité des candidats médicaments, la tuberculose, la neurologie translationnelle, la médecine personnalisée du diabète ou des programmes de formation et de sensibilisation du grand public.

Le projet Novel methods leading to new medications in depression and schizophrenia (NEWMEDS) est un projet IMI associant 19 institutions dans 12 pays, des compagnies pharmaceutiques comme Astra Zeneca, Eli Lilly, Janssen Pharmaceutica, Lundbeck A/S, Novartis, Orion, Pfizer et Servier, ainsi que des PME. ${ }^{[13]}$ Le consortium a déjà rassemblé, en une seule base de données, les données anonymes de 23401 patients ayant participé à 67 essais cliniques qui ont évalué 11 produits dans 25 pays. Ceci constitue la plus grande base de données pour la recherche thérapeutique en psychiatrie.

L'organisation SAGE Bionetworks ${ }^{[14]}$ est une organisation sans but lucratif dont la mission est de créer un bien commun, une plateforme donnant accès à des cartes moléculaires prédictives des maladies. ${ }^{[15]}$ SAGE anticipe la nécessité de mettre en commun les masses d'information issues de l'analyse moléculaire des maladies, en particulier des cancers, pour décrire des modèles moléculaire prédictifs (et pertinents) de ces maladies. Ceci constituera une ressource considérable pour le développement de nouveaux médicaments.

Les projets précompétitifs à succès naissent de la prise de conscience, ensemble, de difficultés et de besoins communs. Le projet de R\&D collaboratif Centre de ressource de modèles expérimentaux de cancer (CReMEC) en est une illustration 
remarquable. Il est né du manque de modèles humains précliniques pertinents de cancer, tant pour comprendre la biologie des cancers que pour valider en préclinique l'efficacité de nouvelles molécules. C'est le premier projet labellisé par le pôle de compétitivité mondial Medicen Paris Region, en 2005. Il a permis de créer collection de plus de 50 modèles de cancers colorectaux humains chez la souris immunodéprimée, complètement caractérisés sur le plan histopathologique, moléculaire et pharmacologique (réponse in vivo aux traitements standard du cancer colorectal). ${ }^{[16]}$ Ces modèles contribuent à accélérer le développement de nouvelles thérapies contre cette maladie. Le programme CReMEC a allié 4 entreprises - Ipsen, Oncodesign (porteur du projet), Sanofi-Aventis, l'Institut de Recherche Servier - et 10 équipes académiques du Cancéropôle Île-de-France issues de l'Assistante publique-Hôpitaux de Paris (AP-HP), du Centre national de la recherche scientifique (CNRS), de l'Institut national de la santé et de la recherche médicale (INSERM), de l'Institut Curie, de l'Institut Gustave-Roussy et de l'Université Paris VII Diderot. La réussite du projet doit beaucoup à la synergie entre les différents partenaires qui ont mutualisé leurs compétences à chaque étape, depuis la collection de la tumeur au bloc opératoire jusqu'à la caractérisation moléculaire et pharmacologique des modèles. Ce projet a été co-financé par l'État (fond unique interministériel) et l'Industrie.

\section{Définition}

À l'issue des trois jours de travaux, les membres de la Table Ronde de Giens, où toutes les parties prenantes étaient présentes, ont proposé une définition.

\subsection{Ce que c'est}

Un projet de recherche précompétitive aborde une thématique précise, avec un objectif défini destiné à résoudre un problème (par exemple «un goulet d'étranglement» dans le domaine du médicament) que l'on ne peut résoudre seul.

Un tel projet a pour vocation de créer des modèles, des outils, des plateformes, de la connaissance.

In fine, c'est favoriser l'émergence de l'innovation sur un lit de connaissances et de plateformes mutualisées.

Un tel projet est déployé par un consortium multidisciplinaire qui s'ouvre à de nouvelles compétences et collaborations.

Les partenaires apportent leur background : "Quand on entre, on partage et on en bénéficie ».

\subsection{Ce que ce n'est pas}

Un projet de recherche précompétitive ne se résume pas à la notion de partenariat public-privé, même si des partenaires publics et des partenaires privés y sont très souvent associés.

Ce n'est pas une collaboration habituelle, sur projet, avec un donneur d'ordre, l'Industriel.

Elle n'est pas définie par la brevetabilité ou la nonbrevetabilité des produits de la recherche.

Il n'y a pas un seul modèle de collaboration précompétitive, mais bien au contraire plusieurs modalités possibles. ${ }^{[17]}$

Enfin, il est essentiel de considérer que la frontière entre compétitif et précompétitif est extrêmement variable, y compris au sein même du consortium, en fonction des partenaires et en fonction du temps. En effet, si le « lit d'innovation» est partagé, il faut rester conscient qu'à un certain moment le compétitif peut reprendre le pas sur le partage complètement ouvert des connaissances, pour des raisons simples de viabilité économique des entreprises et des institutions.

\section{Les facteurs clés de succès}

Des facteurs de succès se dégagent très clairement des exemples de consortium «privé-public » et de programmes cadres européens (ex : IMI) à notre disposition. Parmi les conditions clés pour permettre l'émergence de projets innovants et performants dans le secteur précompétitif et élargir efficacement le champ du mutuellement partagé, figurent la vision commune de l'objectif, la motivation et la qualité du coordinateur et des acteurs impliqués, une gouvernance partagée, une information réciproque, un lien de confiance.

Accepter de comprendre le partenaire avec ses objectifs, de donner, échanger, partager la propriété intellectuelle correspond à un changement culturel nécessaire pour ces projets complexes. L'anticipation des règles de fonctionnement, de décision, de valorisation et de publication permettent cette synergie.

\section{Les menaces}

La lourdeur de la gestion du projet liée au nombre d'intervenants, la fragilité financière ou organisationnelle des petites entreprises, la difficulté de valorisation de ces programmes pour l'évaluation des chercheurs, les ressources financières insuffisantes, la difficulté à anticiper et à résoudre la question de la gestion de la propriété intellectuelle et les conflits d'intérêt non déclarés sont autant de freins rencontrés dans la mise en place des programmes précompétitifs. 


\section{La propriété intellectuelle}

La propriété intellectuelle est un point essentiel qu'il s'agit de partager. Chaque partenaire public ou privé défend une politique de protection de sa propriété intellectuelle qui se veut la plus efficace possible. Si longtemps les chercheurs académiques n'intégraient pas la valorisation dans la déclinaison applicative de leur recherche, la Propriété intellectuelle et la création d'entreprise sont désormais aussi des préoccupations et des objectifs des institutions académiques avec la création par exemple d'INSERM Transfert, d'IGR\&D, de FIST et maintenant les Sociétés d'accélération du transfert de technologies (SATT) et France Brevet. C'est dire que les modalités de gestion de la propriété intellectuelle dans un projet de recherche précompétitive doivent mettre en musique les choix stratégiques et les décisions des partenaires plutôt que guider ces choix et ces décisions.

La propriété intellectuelle (PI), plus spécifiquement dans le domaine de la «pharma» où la mise sur le marché d'un médicament est très longue (en moyenne 15 ans de développement), est toujours un point majeur sur lesquels les industriels s'appuient pour financer leur recherche. En effet, avec une durée de protection du brevet de 20 ans comme dans les autres secteurs, à laquelle peuvent s'ajouter au mieux 5 ans de protection complémentaire et des prix régulés, la rentabilité est très difficilement au rendezvous. Ce phénomène, comme il a été décrit plus haut, s'est accentué. Les breakthrough products qui s'appuient sur une PI forte et sont générateurs de retours financiers importants se font de plus en plus rares.

Depuis quelques années, les industriels ont pris conscience que seule l'innovation pouvait leur rendre cette possibilité mais que, pour avoir une PI forte, il était nécessaire de reconstruire un «terroir » scientifique solide et nouveau sur lequel beaucoup d'acteurs pourraient travailler librement et faire jaillir l'innovation à protéger. Il faut bien noter qu' avoir cette approche «open » ou «précompétitive » n'est pas synonyme d' "open access », la PI restant toujours essentielle. ${ }^{[18]}$ Cependant, c'est plus particulièrement dans les domaines de recherche plus fondamentale que l'ouverture se crée, laissant aux scientifiques la capacité d'être créatifs, la PI étant alors un outil majeur dès que la phase de développement du produit ou de la technologie approche.

Tout naturellement, c'est entre les laboratoires académiques de recherche et les industriels privés que ces relations de partenariats plus spécifiques se sont développées, selon différents types dans lesquels les rôles de chaque partenaire ont évolué. La notion de partage des risques a été intégrée au classique «l'académique publie et l'industriel finance et commercialise », d'où un partage des apports mais aussi des retours plus ouverts.

Au-delà de la notion de partage sur un plan purement technique, s'est ajoutée une notion plus culturelle que décrit S. Friend,
President \& CEO de Sage Bionetworks : «Think of a world where interlab communication is equal to intralab communication ${ }^{.}{ }^{[19]}$ De même, les champs du partage se sont élargis avec une plus grande part d'échange de personnel et d'utilisation de plateformes et matériels. La notion de retour auprès des partenaires a également évolué avec une possibilité plus importante laissée aux académiques de s'appuyer sur des résultats qu'ils n'avaient préalablement pas toujours la liberté d'utiliser (par exemple des cibles, produits « outils de recherche », des modèles créés qui ne sont plus l'exclusivité des industriels ...).

Même si le principe général repose sur un partage en amont de connaissances et un partage en aval des retours s'ils existent, ce type de partenariat stratégique précompétitif est par essence du « sur-mesure » et adapté à la fois au domaine (approche différente dans la microélectronique et dans le vaccin par exemple) et aux types de produits ou technologies concernées. Althshuler a classé ces partenariats en 8 catégories en fonction des résultats qu'ils produisent, des apports et des possibilités d'exploitation. ${ }^{[17]}$

D'une façon générale, il apparait que la mise en place de partenariats précompétitifs doivent impérativement tenir compte, très tôt dans la discussion, des deux notions suivantes :

- Quel est le vrai but et type de la collaboration (développement d'outils, génération de données, création pure de connaissance, ou développement de produit à visée thérapeutique, diagnostic ...)?

- Quelle est la contribution respective des partenaires, à la conception du partenariat et possible dans le futur (apports directs en financement, apport en personnel, en compétences, en plateformes techniques ...)?

Enfin, certaines approches pilotes au sein de certains accords sont plus originales, en laissant des zones libres de propriété intellectuelle ou «IP-Free zones».

Le Biomarker consortium illustre la faisabilité d'une gestion efficace de la propriété intellectuelle dans un projet précompétitif. ${ }^{[20]}$ Il s'agit d'un partenariat public / privé dirigé par the Foundation for the national institutes of health (FNIH). L'objectif est de promouvoir le développement, la qualification et l'acceptabilité d'un point de vue réglementaire des biomarqueurs pour le développement des nouveaux médicaments, la médecine prédictive et les tests diagnostiques. Les projets conduisent à la création d'innovation et de connaissances nouvelles.

Officiellement lancé en octobre 2006, ce consortium a apporté en 2 ans des premiers résultats positifs sur le biomarqueur Adiponectine, montrant clairement qu'après un traitement de 6 à 8 semaines par un récepteur au facteur activé de prolifération des peroxysomes (peroxisome proliferator-activated receptor ou PPAR agoniste) existait une corrélation négative avec l'évolution de l'hémoglobine glycosylée (HbA1c). ${ }^{[21]}$ Seul un regroupement 
d'information et de données de plusieurs acteurs pouvait permettre une telle performance. En effet, des données de 2688 patients diabétiques de type II ont été analysées, dont certaines préexistantes et appartenant à différents groupes publics et privés.

Une des clés majeures de succès a été la mise en commun de données stratégiques issues des différents partenaires avec, pour éviter les problèmes de propriété intellectuelle, la réalisation d'un codage en aveugle, de sorte qu'aucun partenaire ne puisse savoir quelles données propres avaient contribué à l'obtention des résultats. Plus précisément, la charte que doit signer chaque partenaire participant à un projet de recherche au sein du Consortium stipule qu'il donne « une licence d'utilisation gratuite, limitée dans le temps, non exclusive sur les données préexistantes, le matériel et le savoir faire mais pour un usage en recherche uniquement et strictement lié au projet». Il est à noter que certains acteurs, même avec un codage, n'ont pas voulu partager des données stratégiques.

Par ailleurs, les résultats des projets de recherche sont partagés. Plus précisément, les participants à un projet peuvent déposer des brevets sur les résultats générés mais donnent une licence non exclusive et gratuite aux autres membres du projet ainsi qu'un accès gratuit pour utilisation en recherche aux autres membres du consortium.

D'autres règles intéressantes sont à noter comme par exemple la notion de garantie de l'objectivité sur les résultats par l'obtention d'un financement dédié des études statistiques, avant chaque démarrage de projets et d'une réalisation des tests par un acteur extérieur au consortium.

De nouveaux concepts restent à explorer en matière de propriété intellectuelle comme le patent pooling, développé dans le secteur de l'électronique telle que le DVD. Un patent pool est un groupement de brevets qui permet à plusieurs sociétés, personnes ou organisations de mettre en commun des brevets considérés comme indispensables à la mise en oeuvre d'une technologie. Les patent pools, comme tout groupement, permettent de diminuer les coûts des transactions, en proposant de licencier des brevets «en masse » aux membres du groupe. Ainsi, chaque titulaire d'un brevet dont la technologie est nécessaire au fonctionnement d'un produit, met son invention à disposition de tous les membres du groupement. Les brevets apportés au «groupement » sont alors concédés sous forme de licence à chacune des sociétés membres du «groupement», ce qui leur permet d'utiliser la technologie concernée pour ses propres produits, sans faire l'objet de contrefaçon et sans négocier une licence d'exploitation pour chaque produit qu'elle sera amenée à fabriquer. Le principe du patent pool est relativement avantageux, mais il existe une contrepartie à intégrer ce groupement. Dès lors qu'il existe une mutualisation des inventions brevetables, le titulaire des droits de propriété industrielle se voit automatiquement déchu de la gestion exclusive de son monopole. Il ne pourra plus dès lors, négocier des redevances, ni accorder d'autres licences sur son brevet.

En résumé, les principes sur lesquels la protection intellectuelle doit être définie dans les projets pré-compétitifs sont :

- accepter de comprendre les enjeux des partenaires, d'échanger et de partager les résultats antérieurs;

- protéger les résultats obtenus dans le projet, de façon partagée ou non, et les rendre accessibles.

\section{Financements}

Les principes de partage et de mutualisation de la recherche précompétitive conduisent à envisager nécessairement les cofinancements à partir de fonds privés, de fonds publics, voire de mécénat.

En France, il y a beaucoup d'initiatives de financements publics pour faire levier et inciter les investissements privés dans le domaine R\&D, en particulier en Santé. Des changements profonds ont eu lieu au cours des dix dernières années dans le financement public de l'innovation, avec beaucoup d'actions structurantes (pôles de compétitivité, instituts Carnot, Laboratoires d'excellence, Instituts Hospitalo-Universitaires... ). Il y a un fort investissement de l'État pour favoriser les partenariats publicsprivés et la valorisation de la recherche académique même s'il faut regretter la diversité des guichets et la complexité des démarches administratives.

Il n'y a pas en France, pour le moment, de financement fléché sur la recherche précompétitive mais un grand nombre d'appels à projets sont ouverts à ce type de recherche. Faut-il en créer un?

Seule la plateforme Innovative medicine initiative codéveloppée par l'Europe et l'EFPIA est entièrement dédiée à la recherche précompétitive, publique-privée, pour résoudre des goulets d'étranglement identifiés par les industriels du médicament.

\section{Conclusion}

Au-delà du terme «précompétitif » il s'agit de développer une recherche en partenariat fondée sur des principes nouveaux :

- comprendre et partager;

- intégrer la complexité du vivant;

- agir dans l'interdisciplinarité.

C'est un changement culturel profond, pour tous les acteurs, mais nécessaire pour améliorer les connaissances sur les maladies et optimiser le développement des médicaments afin que le patient puisse en bénéficier in fine. 


\section{Recommandations de la Table Ronde}

1. Ouvrir le domaine à de nouvelles compétences et favoriser l'interdisciplinarité.

Actions :

- disséminer vers l'Alliance pour la recherche et l'innovation des industries (ARIIS) et l'alliance pour les sciences de la vie et de la santé (AVIESAN);

- proposer une Table Ronde de Giens avec mathématiciens sur sujet dédié.

2. Identifier les nouveaux thèmes relevant de la recherche précompétitive dans le domaine du médicament et du dispositif médical.

3. S'inspirer de l'expérience émanant d'autres secteurs industriels.

\section{Participants}

Olivier Amedee-Manesme (Paris Biotech Santé), Zeina Antoun (GlaxoSmithKline), Olivier Arnaud (Inserm Transfert, Paris), Antoine Audry (Medtronic), Alexandra Auffret (CICCPECT, Marseille), Olivier Blin (CIC UPCET, Marseille), Antoine Bril (Institut de recherches Servier), Pierre Braquet (OncoLogics), Soizic Courcier (GlaxoSmithKline), Cécile Delval (Institut Pasteur, Paris), Jacques Demotes Mainard (Ministère de l'Enseignement Supérieur et de la Recherche), Isabelle Diaz (Leem), Elisabeth Fery Lemonier (Fédération Hopitalière de France, Paris), Isabelle Giri (Access Conseil, Verrières le Buisson), Valérie Izard (Haute Autorité de Santé, Saint Denis la Plaine), Valéry Labonne (CrossWorlds HealthCare Professionals, Paris), Olivier Louvet (Ministère de la Santé, Paris), Carole Neves (Sanofi Aventis), Isabelle Pelletier Bressac (Institut Gustave Roussy, Villejuif), Yannick Pletan (Roche), Sophie Ravoire (Lilly), Pierre Schiavi (Servier), Daniel Vasmant (Ministère de l'Economie, des Finances et de l'Emploi, Paris), Gilles Vassal (Institut Gustave Roussy, Villejuif)

\section{Références}

1. Munos B. Lessons from 60 years of pharmaceutical innovation. Nat Rev Drug Discov 2009 Dec;8(12): 959-68

2. Eichler HG, Aronsson B, Abadie E, et al. New drug approval success rate in Europe in 2009. Nat Rev Drug Discov 2010 May 9(5), 355-6
3. Barter PJ, Caulfield M, Eriksson M, et al. Effects of torcetrapib in patients at high risk for coronary events. N Engl J Med 2007 Nov 22; 357(21): 210922

4. Learning lessons from Pfizer's $\$ 800$ million failure. Nat Rev Drug Discov 2011 Mar;10(3): 163-4

5. Woodcock J. Precompetitive research: a new prescription for drug development? Clin Pharmacol Ther 2010 May; 87(5): 521-3

6. Paul SM, Mytelka DS, Dunwiddie CT, et al. How to improve R\&D productivity: the pharmaceutical industry's grand challenge. Nat Rev Drug Discov 2010 Mar; 9(3): 203-14

7. Nathan C. Aligning pharmaceutical innovation with medical need. Nat Med 2007 Mar; 13(3): 304-8

8. Grabowski H, Vernon J, DiMasi JA. Returns on research and development for 1990s new drug introductions. PharmacoEconomics 2002; 20(Suppl 3): $11-29$

9. http://www. corem.qc.ca

10. Carayannis EG, Alexander J. Strategy, structure, and performance issues of precompetitive R\&D consortia: insights and lessons learned from SEMATECH. Engineering Management IEEE Transactions on May 2004; 51 (2): 226-32

11. http://www.tifn.nl

12. http://www.alzheimer-europe.org/FR/Research/PharmaCog

13. http://newmeds-europe.com

14. http://www. sagebase.org

15. Derry JMJ, Mangravite LM, Suver C, et al. Developing predictive molecular maps of human disease through community-based modeling. Nature Precedings April 2011 http: //precedings.nature.com/documents/ 5883/version/1/files/npre20115883-1.pdf

16. Julien S, Merino-Trigo A, Lacroix L, et al. CReMEC initiative: characterization of patient-derived colorectal tumor models and correlation with patient profile. Proc Am Assoc Cancer Res, 2010 abst. 4169 http: //www . oncodesign. fr/posters/2010/AACR2010CReMEC.pdf

17. Altshuler JS, Balogh E, Barker AD, et al. Opening up to precompetitive collaboration. Sci Transl Med 2010 Oct 6; 2(52): $52 \mathrm{~cm} 26$

18. Melese T, Lin SM, Chang JL, et al. Open innovation networks between academia and industry: an imperative for breakthrough therapies. Nat Med 2009 May; 15(5): 502-7

19. Friend SH. The need for precompetitive integrative bionetwork disease model building clinical pharmacology \& therapeutics. Clin Pharmacol Ther 2010; 87(5): 536-9

20. http: //www. biomerkersconsortium.org

21. Wagner JA, Prince M, Wright EC, et al. The Biomarkers consortium: practive and pitfalls of ope-source precompetitive collaboration. Clin Pharmacol Ther 2010; 87: 539-42

Correspondance et offprints : Gilles Vassal, Direction de la Recherche Clinique, Institut Gustave Roussy, 114 rue Edouard Vaillant, 94805 Villejuif Cedex, France.

E-mail : gilles.vassal@igr.fr 